\title{
Prevalência e Fatores Associados à Síndrome de Burnout em Psicólogos
}

\section{PREVALENCE AND FACTORS ASSOCIATED WITH THE BURNOUT SYNDROME AMONG PSYCHOLOGISTS}

\author{
Sandra Yvonne Spiendler Rodriguez', Mary Sandra Carlotto²
}

1. Pontifícia Universidade Católica do Rio Grande do Sul, Porto Alegre, Brasil. Bolsista CAPES.

2. Pontifícia Universidade Católica do Rio Grande do Sul, Porto Alegre, Brasil.

\begin{abstract}
RESUMO
Este estudo avaliou a prevalência e os fatores associados à Síndrome de Burnout em uma amostra de 518 psicólogos que atuam no Rio Grande do Sul/Brasil. Foram utilizados como instrumentos de pesquisa o Cuestionario para la Evaluación del Síndrome de Quemarse por el Trabajo - CESQT- e um questionário para as variáveis sociodemográficas e laborais. Os resultados evidenciam uma prevalência de 7,5\% de psicólogos que apresentam o Perfil 1 de Burnout e 9,8\% o Perfil 2. Apresentam maior risco de desenvolvimento da Sindrome de Burnout os homens, sem companheiro(a) fixo(a), sem filhos, com menores ganhos financeiros, menor carga horária de trabalho semanal e maior número de atendimentos/dia, os que atuam em atividades além da Psicologia ou que atuam em apenas uma área da Psicologia, os que mantém vínculo empregatício e não participam de grupos de estudo ou associações da Psicologia. Aponta-se a necessidade de intervenções de prevenção e reabilitação.
\end{abstract}

(Rodriguez S, Carlotto M, 2014. Prevalência e Fatores Associados à Síndrome de Burnout em Psicólogos. Cienc Trab. Sep-Dic; 16 [51]: 170-176).

Palavras-chave: SÍNDROME DE BURNOUT; PSICÓLOGOS; SAÚDE OCUPACIONAL; ESTRESSE OCUPACIONAL.

\section{ABSTRACT}

This study evaluated the prevalence and the factors associated with the Burnout Syndrome in a sample of 518 psychologists working in the state of Rio Grande do Sul (Brazil). The Cuestionario para la Evaluación del Síndrome de quemarse por el Trabajo - CESQT - and a questionnaire for demographic and labor variables were used as research instruments. Results show a 7,5\% prevalence of psychologists with Profile 1 of Burnout and 9,8\% prevalence with Profile 2. Men, professionals without a steady partner, people who have no children, professionals with smaller financial gains, lower weekly working hours and a greater number of counseling sessions/day, those engaged in activities beyond Psychology or those who work with only one area of Psychology, and those who are employed and do not participate in study groups or psychological associations face greater risks of developing Burnout Syndrome. This study points to the need to promote interventions of prevention and rehabilitation.

Key words: BURNOUT SYNDROME; PSYCHOLOGISTS; OCCUPATIONAL HEALTH; OCCUPATIONAL STRESS.

\section{INTRODUÇÃO}

\section{Síndrome de Burnout}

A Síndrome de Burnout (SB) é um fenômeno psicossocial presente na sociedade contemporânea, a qual se encontra permeada de diversas transformações econômicas, políticas e tecnológicas que têm tensionado as relações de trabalho e laboral. ${ }^{1}$ Resulta de um estado de estresse crônico, típico do cotidiano do mundo do trabalho $^{2}$ e, diretamente relacionado aos estressores interpessoais

Correspondencia / Correspondence:

Sandra Yvonne Spiendler Rodriguez

e-mail: psicologa07@gmail.com

Mary Sandra Carlotto

e-mail: mscarlotto@pesquisador.cnpq.br

Av. Ipiranga, 6681. Partenon Porto Alegre /RS

Tel.: (51) 33203500

Recibido: 15 de Agosto de 2014 / 23 de Septiembre DE 2014 que afetam profissionais que mantém contato direto, frequente e emocional com os clientes atendidos. ${ }^{3,4}$ A SB tem sido apontada como um importante problema de saúde pública, dadas suas consequências negativas ${ }^{5}$, destacando-se as individuais, como os adoecimentos físicos e mentais, e as organizacionais, como o aumento do absenteísmo e da rotatividade que implicam em queda da produtividade e qualidade. ${ }^{6}$

Segundo o modelo teórico proposto por Gil-Monte ${ }^{7}$, a SB é um tipo de adoecimento ocupacional constituído por quatro dimensões: 1) Ilusão pelo trabalho, que indica o desejo individual para atingir metas relacionadas ao trabalho, sendo estas percebidas pelo sujeito como atraentes e fonte de satisfação pessoal; 2) Desgaste Psíquico, caracterizado pelo sentimento de exaustão emocional e física em relação ao contato direto com pessoas que são percebidas como fonte causadora de problemas; 3) Indolência, evidencia da presença de atitudes de indiferença junto às pessoas que necessitam serem atendidas no ambiente de trabalho; 4) Culpa, definida pelo surgimento de sentimentos de culpabilização por atitudes e comportamentos adotados não condizentes com as normas internas e cobrança social acerca do papel profissional. No modelo desenvolvido pelo autor é possivel distinguir dois perfis de Burnout. 0 perfil 1, derivado de um conjunto de senti- 
mentos e condutas ligadas ao estresse laboral originando uma forma moderada de mal-estar, mas não impossibilitando o profissional de exercer suas atividades laborais mesmo que pudesse realizá-las de forma mais eficaz. 0 perfil 2, constitui-se de casos clínicos mais deteriorados, incluindo os sintomas já apresentados, acrescido do sentimento de culpa.

\section{Síndrome de Burnout em Psicólogos}

0 psicólogo é um profissional que atua essencialmente na promoção da saúde mental e qualidade de vida das pessoas. Seu cotidiano laboral envolve o manejo dos problemas de origem psicoafetiva das pessoas $^{8}$, o que pressupõe cuidado e atenção com o outro. Portanto, necessita estabelecer uma relação emocional próxima com as pessoas atendidas, da qual decorrem demandas emocionais intensas, por vezes, excessivas, o que pode tornar o psicólogo susceptivel ao Burnout. ${ }^{9} \mathrm{Na}$ atualidade, a essa demanda emocional, soma-se à laboral, uma vez que cresce o número de profissionais que atuam em diferentes áreas e com mais de um vinculo de trabalho. ${ }^{10}$

A literatura internacional tem se mostrado interessada na investigação de Burnout em psicólogos, considerando sua prevalência e fatores associados. Quanto à prevalência, os resultados variam desde a não identificação de Burnout ${ }^{11}$ até estudos que indicam uma prevalência de $20 \%$. $^{12}$

No que diz respeito aos fatores associados, identifica-se relação com fatores sociodemográficos e laborais. Quanto aos sociodemográficos, verificam-se em alguns estudos que os homens apresentam niveis mais elevados de despersonalização ${ }^{13} \mathrm{e}$, as mulheres maior exaustão emocional. ${ }^{14}$ Quanto à idade, estudo de Ruppert e Kent ${ }^{15}$ indica que quanto maior a idade menor a exaustão emocional e a despersonalização e maior o sentimento de realização pessoal no trabalho.

No que concerne as laborais, estudos revelam que o aumento da carga horária de trabalho ${ }^{12,15,16}$ e do envolvimento com demandas burocráticas e administrativas ${ }^{15,17}$ associam-se a exaustão e despersonalização. 0 controle sobre o trabalho, possivel na atividade profissional autônoma, associa-se a realização pessoal. ${ }^{12,13,15,17}$ Profissionais que exercem atividades em instituições públicas ${ }^{14} \mathrm{e}$ no sistema prisional ${ }^{18}$, experimentam altos níveis de exaustão e despersonalização.

Embora seja possivel identificar um importante incremento de estudos de Burnout ao longo dos anos e sua crescente ampliação do foco de pesquisa com variadas profissões, incluindo médicos, enfermeiros, assistentes sociais, policiais, bombeiros, professores entre outros $^{19}$, ainda são relativamente escassos, no Brasil, os estudos com psicólogos. ${ }^{8,20}$ Estes, quando estudados, são habitualmente incluídos como parte da categoria denominada de profissionais da saúde. ${ }^{20,21}$ Estudos de prevalência e fatores associados são um importante passo para auxiliar no diagnóstico das doenças relacionadas ao trabalho ${ }^{8}$, pois embora a SB já seja reconhecida no Brasil pelo Manual das Doenças Relacionadas ao Trabalho ${ }^{22}$, sua prevalência e fatores de risco ainda são pouco conhecidos. ${ }^{23}$ Pelo exposto, o presente objetivou avaliar a prevalência e os fatores sociodemográficos e laborais associados ao Burnout em uma amostra de psicólogos.

\section{MÉTODO}

\section{Participantes}

A amostra foi constituída por 518 psicólogos que atuam no estado do Rio Grande do Sul/Brasil. Foi estabelecido como critério de inclusão ter mais de um ano de exercício profissional. A maioria dos participantes é do sexo feminino (77,6\%), sem filhos (57,5\%), com união estável (60,2\%). A idade média dos profissionais é de 34,7 anos (DP $=7,79$ ) e possuem de 1 a 40 anos de atuação profissional $(\mathrm{M}=10,6 ; \mathrm{DP}=8,27)$. Quanto a remuneração, a maioria possui ganhos financeiros entre 3 e 6 salários mínimos (51,6\%). No que se refere a área de atuação, a maioria exerce suas atividades na área Clínica $(61,4 \%)$, seguida da Psicologia Organizacional e do Trabalho (37,6\%), Comunitária (12,9), Hospitalar (9,7\%), Escolar (9,3\%), Jurídica (6,9\%), Trânsito (2,1\%) e Esporte (0,8\%). Quanto à formação, 50,5\% dos psicólogos possuem formação em nível de especialização e 24,3\%, em nível de mestrado e/ou doutorado.

\section{INSTRUMENTOS}

Os dados foram coletados por meio dos seguintes instrumentos autoaplicáveis: 1. Questionário de dados sociodemográficos (sexo, idade, situação conjugal, filhos, remuneração) e laborais (formação, tempo de experiência profissional, área(s) de atuação na psicologia, tipo de vínculo empregatício, possuir outra atividade profissional, carga horária semanal de trabalho, quantidade de pessoas atendidas, satisfação com o trabalho, pensarem trocar de profissão). 2. Cuestionario para la Avaluación del Síndrome de Quermarse por el Trabajo (CESQT), versão adaptada para o Brasil por Gil-Monte, Carlotto e Câmara. ${ }^{24}$ Trata-se de um instrumento constituído de 20 itens que se distribuem em quatro subescalas: Ilusão para o trabalho ( 5 itens, alfa=0,72); Desgaste psíquico ( 4 itens, alfa=0,86); Indolência (6 itens, alfa=0,75); e, Culpa ( 5 itens, alfa=0,79). Os itens são avaliados com uma escala tipo Likert de frequência de cinco pontos, na qual utiliza-se o 0 para "nunca", 1 para "raramente", 2 para "às vezes" 3 para "frequentemente" e, 4 para "muito".

\section{Procedimentos de coleta dos dados}

Os dados do estudo foram coletados por meio de pesquisa on-line mediante convite via e-mail. Para fins de recrutamento dos participantes, foi utilizada a técnica do Respondent Driven Sampling (RDS) que combina a amostragem em bola de neve (Snow Ball Sampling ${ }^{25}$ e a utilização de redes sociais, como uma forma de compensar a amostragem não-aleatória. ${ }^{26}$ Num primeiro momento, identificaram-se as sementes da amostra, ou seja, os indivíduos que pertenciam a população alvo do estudo e, que poderiam recrutar novos participantes. Assim, os primeiros participantes, constituídos como a $1^{\mathrm{a}}$ onda, foram psicólogos que tiveram seus e-mails mapeados de fontes públicas como sites de associação associações, fundações, sindicatos e demais órgãos representativos da Psicologia. A $2^{\text {a }}$ onda foi realizada por meio da divulgação do Conselho Regional de Psicologia e e do Rio Grande do Sul e nas redes sociais de profissionais que compõem a rede de contato das pesquisadoras. A $3^{\text {a }}$ onda foi desenvolvida por meio do mapeamento de guias telefônicos. ${ }^{27,26} 0$ estudo foi aprovado pelo Comitê de Ética em Pesquisa da PUC/RS sob o Parecer $n^{\circ} 445847$.

\section{Procedimentos de análise dos dados}

Os dados foram registrados e analisados no Pacote Estatístico para Ciências Sociais (SPSS-Statistical Package of Social Science, versão 17.0). Para a identificação dos níveis da SB, foram utilizadas as orientações do Manual do CESQT. ${ }^{28}$ Para determinar a prevalência da SB da amostra, foram calculados os percentis a partir das pontuações brutas, considerado o percentil $\geq$ P90 como de risco de Burnout. 
Foram considerados Perfil 1 os casos que apresentaram pontuações $\geq$ P90 na pontuação média dos 15 itens que formam as subescalas de Ilusão pelo trabalho (invertida), Desgaste psíquico e Indolência, mas inferiores ao P90 na subescala de Culpa. Para o cálculo do Perfil 2 foram incluídos os casos identificados de Perfil 1 , acrescido dos $\geq$ ao P90 da subescala Culpa.

Inicialmente realizaram-se análises descritivas de caráter exploratório a fim de avaliar a distribuição dos itens, casos omissos e identificação de extremos. A análise dos dados foi realizada por meio da prova de correlação de Pearson (força e magnitude da associação entre variáveis contínuas), t de student (comparação de médias de duas variáveis categóricas), considerando um nível de significância de 5\%. 0 tamanho do efeito foi calculado pela diferença média padronizada entre dois grupos ( $d$ de Cohen), que considera 0,2 um valor indicativo de um efeito pequeno, 0,5 um efeito médio e 0,8 um tamanho de efeito grande29 e pelos coeficientes de regressão padronizados (R2) calculados para cada correlação significativa e de acordo com Field. ${ }^{30}$

\section{RESULTADOS}

As dimensões do CESQT apresentaram adequados valores de confiabilidade dos resultados, considerando-se resultados $\alpha \geq$ $0,70 .{ }^{31}$ Em relação às médias das dimensões, o valor mais alto foi obtido para a Ilusão pelo trabalho $(\mathrm{M}=2,96)$ e o mais baixo para a Culpa $(\mathrm{M}=0,97)$. No caso da dimensão Ilusão pelo trabalho, as questões estão formuladas em sentido positivo, de forma que altas pontuações nessa dimensão revelam baixos níveis de SB (Tabela 1). No presente estudo, encontrou-se uma prevalência de 11,6\% dos participantes com sentimentos de baixa Ilusão pelo trabalho, 26,1\% com alto nível de Desgaste psíquico, 11,2\%, de Indolência e 10,8\% de Culpa. Quanto aos perfis de Burnout, identificou-se uma prevalência de casos no Perfil 1 da SB de 7,5\% e 9,8\% de Perfil 2 (Tabela 2).
Tabela 1.

Médias, desvio padrão e consistência interna das dimensões de CESOT.

$\begin{array}{lccc}\text { Variáveis } & \mathrm{M} & \mathrm{DP} & \alpha \\ \text { Ilusão pelo trabalho } & 2,96 & 0,71 & 0,87 \\ \text { Desgaste psíquico } & 2,03 & 0,88 & 0,84 \\ \text { Indolência } & 1,11 & 0,57 & 0,78 \\ \text { Culpa } & 0,97 & 0,61 & 0,82\end{array}$

Tabela 2.

Número e porcentagem de psicólogos com níveis críticos de SB de acordo com os percentis (P) do Manual do CESOT.

$\begin{array}{lcc}\text { Variáveis } & P<90 & P \geq 90 \\ \text { llusão pelo trabalho } & 458(88,4 \%) & 60(11,6 \%) \\ \text { Desgaste psíquico } & 383(73,9 \%) & 135(26,1 \%) \\ \text { Indolência } & 460(88,8 \%) & 58(11,2 \%) \\ \text { Culpa } & 462(89,2 \%) & 56(10,8 \%) \\ \text { Perfil 1 } & 479(92,5 \%) & 39(7,5 \%) \\ \text { Perfil 2 } & 467(90,2 \%) & 51(9,8 \%)\end{array}$

A análise da relação entre variáveis sociodemográficas e laborais qualitativas e as dimensões de Burnout revelam que mulheres apresentam índice mais elevado de Ilusão pelo trabalho que homens e estes, maior Desgaste psíquico. Participantes que possuem companheiro apresentam maior Ilusão pelo trabalho e os que possuem filhos, menor Indolência e Culpa. Os participantes que realizam outra atividade profissional não vinculada à Psicologia apresentam menos Ilusão e maior Desgaste Psíquico. Profissionais que atuam em mais de uma área da Psicologia possuem maior Ilusão e menor Desgaste Psíquico, Indolência e Culpa e, os que trabalham com vínculo empregatício apresentam maior índice de Desgaste Psíquico, Indolência e Culpa. Os psicólogos que frequentam grupos de estudo ou associação possuem maior Ilusão pelo trabalho e menor Desgaste Psíquico, Indolência e Culpa. 0 tamanho do efeito, calculado pelo d de Cohen para os resultados significativos, variou de $-0,18$ (efeito pequeno) a 0,71 (efeito médio) de acordo com os parâmetros determinados por Cohen (Tabela 3). ${ }^{29}$

Tabela 3. Relação entre as dimensões de Burnout e as variáveis qualitativas.

\begin{tabular}{|c|c|c|c|c|c|c|c|c|c|c|c|c|c|c|c|c|}
\hline \multirow[t]{2}{*}{ Variáveis } & \multicolumn{3}{|c|}{ IL } & \multicolumn{5}{|c|}{ DP } & \multicolumn{3}{|c|}{ IN } & \multicolumn{5}{|c|}{$\mathrm{CL}$} \\
\hline & $M$ & DP & $p$ & $d$ & $M$ & DP & $p$ & $d$ & $M$ & DP & $p$ & $d$ & $M$ & DP & $p$ & $d$ \\
\hline \multicolumn{17}{|l|}{ Sexo } \\
\hline Fem & 3,01 & 0,71 & $0,01^{*}$ & 0,30 & 1,91 & 0,86 & $0,01^{*}$ & $-0,57$ & 1,08 & 0,57 & 0,60 & & 0,98 & 0,60 & 0,58 & \\
\hline Masc & 2,80 & 0,67 & & & 2,41 & 0,88 & & & 1,20 & 0,60 & & & 0,94 & 0,63 & & \\
\hline \multicolumn{17}{|c|}{ Companheiro } \\
\hline Sim & 2,87 & 0,69 & $0,03^{*}$ & 0,35 & 2,13 & 0,84 & 0,80 & & 1,18 & 0,51 & 0,37 & & 1,06 & 0,55 & 0,76 & \\
\hline Não & 2,63 & 0,66 & & & 2,30 & 0,73 & & & 1,12 & 0,53 & & & 0,94 & 0,56 & & \\
\hline \multicolumn{17}{|l|}{ Filhos } \\
\hline Sim & 3,03 & 0,70 & 0,07 & & 1,96 & 0,96 & 0,10 & & 1,04 & 0,56 & $0,01^{*}$ & $-0,21$ & 0,91 & 0,56 & $0,04^{*}$ & $-0,18$ \\
\hline Não & 2,92 & 0,71 & & & 2,08 & 0,82 & & & 1,16 & 0,57 & & & 1,02 & 0,64 & & \\
\hline \multicolumn{17}{|c|}{ Outra atividade profissional } \\
\hline Sim & 2,80 & 0,71 & $0,02^{*}$ & $-0,31$ & 2,23 & 0,90 & $0,02^{*}$ & 0,30 & 1,13 & 0,56 & 0,60 & & 1,00 & 0,65 & 0,49 & \\
\hline Não & 3,02 & 0,70 & & & 1,96 & 0,87 & & & 1,10 & 0,58 & & & 0,96 & 0,59 & & \\
\hline \multicolumn{17}{|c|}{ Trabalha mais de uma área } \\
\hline Sim & 3,22 & 0,61 & $0,01^{*}$ & 0,66 & 1,88 & 0,91 & $0,01^{*}$ & $-0,30$ & 0,99 & 0,54 & $0,01^{*}$ &,- 037 & 0,86 & 0,55 & $0,01^{*}$ &,- 032 \\
\hline Não & 2,78 & 0,72 & & & 2,14 & 0,85 & & & 1,20 & 0,58 & & & 1,05 & 0,63 & & \\
\hline \multicolumn{17}{|l|}{ Vínculo } \\
\hline Sim & 2,87 & 0,71 & 0,19 & & 2,16 & 0,86 & $0,01^{*}$ & 0,33 & 1,28 & 0,58 & $0,01^{*}$ & 0,55 & 1,07 & 0,60 & $0,04^{*}$ & 0,30 \\
\hline Não & 2,96 & 0,71 & & & 1,87 & 0,89 & & & 0,97 & 0,55 & & & 0,89 & 0,60 & & \\
\hline \multicolumn{17}{|c|}{ Participa grupo estudo/associação } \\
\hline Sim & 3,26 & 0,56 & $0,01^{*}$ & 0,71 & 1,86 & 0,94 & $0,01^{*}$ & $-0,29$ & 1,01 & 0,56 & $0,02^{*}$ & 0,28 & 0,90 & 0,61 & $0,03^{*}$ & $-0,18$ \\
\hline Não & 2,80 & 0,73 & & & 2,12 & 0,84 & & & 1,17 & 0,58 & & & 1,01 & 0,60 & & \\
\hline
\end{tabular}

Nota: * Diferença significativa ao nível de 5\%; IL: llusão pelo trabalho; DP: Desgaste psíquico IN: Indolência CL: Culpa. 
Resultados obtidos na associação entre variáveis sociodemográficas e laborais quantitativas revelam que quanto maior a renda, a carga horária semanal realizada, a satisfação com a profissão e quanto menos frequente o pensamento em mudar de profissão, maior é a Ilusão pelo trabalho. 0 Desgaste psíquico aumenta na medida em que diminui a renda, a satisfação e com o aumento da quantidade de pessoas atendidas e do pensamento em mudar de profissão. Com relação à Indolência, pode-se verificar que quanto maior a renda e a satisfação com a profissão, menor a Indolência e, na medida em que se eleva a carga horária semanal e o pensamento de mudar de profissão, maior o sentimento de distanciamento com a clientela. 0 sentimento de Culpa diminui com a elevação da renda, com o aumento da satisfação com a profissão e aumenta com a tendência em pensar em mudar de profissão. Os resultados revelam um poder de efeito predominantemente entre pequeno $(R 2=0,01)$ e elevado $(R 2=0,26)$, de acordo com os parâmetros recomendados por Field ${ }^{30}$ (Tabela 4).

\section{Tabela 4.}

Relação entre as dimensões do Burnout e as variáveis quantitativas.

$\begin{array}{lllllllll}\text { Variáveis } & \mathbb{I L} & \mathrm{R}^{2} & \mathrm{DP} & \mathrm{R}^{2} & \mathrm{IN} & \mathrm{R}^{2} & \mathrm{CL} & \mathrm{R}^{2} \\ \text { Idade } & 0,09 & & -0,03 & & -0,01 & & 0,05 \\ \text { Tempo Formação } & 0,07 & & -0,05 & & -0,04 & & -0,00 \\ \text { Renda } & 0,27^{* *} & 0,07 & -0,19^{* *} & 0,04 & -0,12^{* *} & 0,01 & -0,10^{*} & 0,01 \\ \begin{array}{l}\text { Pessoas atendidas } \\ \text { CH seminal }\end{array} & 0,00 & & 0,11^{*} & 0,01 & 0,07 & & 0,01 \\ \begin{array}{l}\text { Satisfação com } \\ \text { a profissão }\end{array} & 0,18^{* *} & 0,03 & 0,06 & & 0,10^{*} & 0,01 & 0,02 \\ \begin{array}{l}\text { Pensar em trocar } \\ \text { de profissão }\end{array} & 0,45^{* *} & 0,20 & -0,39^{* *} & 0,15 & -0,26^{* *} & 0,07 & -0,20^{* *} 0,04 \\ & -0,49^{* *} & 0,24 & 0,40^{* *} & 0,26 & 0,27^{* *} & 0,08 & 0,18^{* *} 0,03\end{array}$

Nota: ${ }^{*}<<0,05 *{ }^{*} p<0,01$

IL: Ilusão pelo trabalho DP: Desgaste psiquico IN: Indolência CL: Culpa.

\section{DISCUSSÃO}

0 objetivo deste estudo foi verificar a prevalência e os fatores associados em uma amostra de psicólogos. Considerando a escala de pontuação utilizada (0-4 pontos), verifica-se que a dimensão de Ilusão pelo trabalho foi a que atingiu a maior média $(M=2,96)$ indicando que os participantes "frequentemente" avaliam que têm conseguido atingir metas profissionais e que essas têm sido percebidas como uma importante fonte de realização profissional. Tal resultado pode revelar que essa dimensão possa estar funcionando como um fator de proteção frente ao desenvolvimento da SB, uma vez que o trabalho foi avaliado "algumas vezes" $(\mathrm{M}=2,03)$ como desgastante. Resultado semelhante foi obtido em professores $^{32} \mathrm{e}$ em profissionais da saúde. ${ }^{21}$

Em relação à Indolência, os participantes "raramente" $(M=1,11)$ sentem que se distanciam afetivamente da sua clientela, resultado também encontrado por Jacques et al. ${ }^{8}$ Segundo os autores, os psicólogos são, dentre os profissionais da saúde, os que apresentam menor insensibilidade e afastamento emocional, provavelmente porque tal comportamento implicaria na presença de um paradoxo profissional, visto que o estabelecimento do vínculo afetivo é um componente fundamental no trabalho dos profissionais. Resultado semelhante foi obtido na dimensão de Culpa $(\mathrm{M}=0,97)$ indicando que os profissionais percebem somente "raramente" suas ações podem não estar correspondendo às expectativas e normas sociais acerca do seu papel profissional.

Considerando-se os critérios de classificação da $\mathrm{SB}^{28}$, foram detectados 90 participantes que poderiam ser considerados casos de SB, 7,5\% (Perfil 1) e 9,8\% (Perfil 2), sendo que neste último incluem-se os casos mais graves, com maior risco de afastamento e incapacitação para o trabalho. Ressalta-se, no entanto, que, para fins de diagnóstico, a SB deve ser confirmada mediante a realização de entrevistas clínicas., ${ }^{7,33}$

Estudo realizado por Tajera e Gómez ${ }^{34}$, com médicos psiquiatras, revelou uma prevalência de 10\% dos profissionais no Perfil 1 e 0,8\% no Perfil 2. Marucco, Gil-Monte e Flamenco ${ }^{33}$ encontraram uma prevalência de 27,64\% de Perfil 1 e 3,25\% de Perfil 2, na avaliação de pediatrias de hospitais. Já, em estudo realizado com profissionais que atuam em centros de atenção psicossocial, Faúndez e Gil-Monte ${ }^{1}$, identificaram uma prevalência de 5,9\% de Perfil 1 e 1,27\% de Perfil 2. Assim, verifica-se que os psicólogos são os que apresentam percentual mais elevado de Perfil 2, dentre outros profissionais da área da saúde, sugerindo que a presença do adoecimento possa estar relacionada às próprias características da profissão, cujo viés vocacional voltado para a ajuda pode incrementar o sentimento de culpa derivado da cobrança social da profissão, tendo em vista que, muitas vezes, atribuem-se aos profissionais da psicologia um poder de cura e resolução de problemas. $^{35}$

0 resultado é preocupante considerando que os profissionais participantes do estudo se encontram em atividade profissional, caracterizando a existência do presenteísmo ocupacional na psicologia. A persistência na realização das funções laborais, mesmo diante da evidência de adoecimento, pode agravar as condições de saúde dos profissionais e repercutir negativamente na qualidade do atendimento prestado. ${ }^{4}$

A associação entre as variáveis sociodemográficas e laborais encontradas neste estudo reforça a compreensão do Burnout como sendo um fenômeno complexo e resultante da interação de múltiplas dimensões, como as individuais ${ }^{36}$ e laborais. ${ }^{3}$ No que se refere às variáveis sociodemográficas, os resultados deste estudo apontam que os homens apresentam maior desgaste psicológico e mulheres, maior ilusão pelo trabalho. Estudos que avaliaram psicólogos americanos revelaram que as mulheres apresentam mais exaustão emocional que os homens $1^{4,15,17}$, revelando resultados contrários aos encontrados neste estudo.

As variações nos resultados entre homens e mulheres podem ser compreendidas à luz do processo de socialização de gênero e do processo de formação. ${ }^{37}$ As mulheres, socializadas para a função de cuidado e atenção das pessoas ${ }^{38,39}$, sentem-se provavelmente confortáveis diante de funções de auxílio e de apoio emocional, o que favorece o exercício da profissão. Por outro lado, aos homens atribuem-se características como praticidade e objetividade ${ }^{40}$, independência, agressividade, dominância, competitividade ${ }^{41}$, de forma que o exercício de funções com cargas emocionais pode exigir um esforço maior, fator este que pode explicar o maior desgaste. No que se refere à formação, embora possa ser esperado que na profissão do psicólogo essas diferenças de gênero socialmente constituídas sejam atenuadas durante a graduação, considerando que esta capacita basicamente para demandas de natureza psicoafetiva, os resultados deste estudo, levam a crer que a formação não é suficiente para produzir mudanças nesse sentido, reforçando que ela é um importante pilar na identidade profissional dos psicólogos sem, contudo, ser a única via de influência na preparação dos profissionais. ${ }^{42}$

Resultados obtidos quanto à situação conjugal revelam que os participantes com companheiro fixo sentem-se mais realizados no trabalho. Esse resultado pode ser entendido a partir da perspectiva 
do apoio social. ${ }^{3}$ É possivel pensar que o companheiro possa auxiliar a atenuar a presença dos estressores decorrentes do trabalho e fortalecer o profissional na busca do alcance de suas metas laborais, na medida em que com ele é possivel compartilhar problemas e frustrações.

Os resultados revelam que profissionais que possuem filhos apresentam menos distanciamento e indiferença em relação às pessoas atendidas no trabalho e sentem-se menos culpados por apresentarem atitudes incompatíveis com o esperado no exercício da profissão de psicólogo, vão ao encontro da literatura que aponta que pessoas com filhos apresentam menores escores de Burnout que pessoas sem filhos. ${ }^{37,43,44}$ Segundo estes estudos, o exercício da maternidade e da paternidade pode fortalecer a preparação dos profissionais, na medida em que a vivência dos problemas e estressores naturalmente presentes na educação dos filhos proporciona experiência e manejo no cuidado com o outro. Assim, é possivel que os profissionais possam desenvolver maior tolerância no manejo dos estressores presentes no trabalho.

No que se refere às variáveis laborais, realizar outra atividade profissional não relacionada à Psicologia aumenta o desgaste psicológico e diminui o sentimento de ilusão pelo trabalho. Resultado semelhante foi identificado por Rupert e Morgan ${ }^{17}$ indicando que o aumento de demandas com atividades que não fazem parte do trabalho específico do psicólogo, diminui a realização profissional. 0 exercício da Psicologia como profissão está associada, segundo Magalhães, Straliotto, Keller e Gomes ${ }^{45}$, com o desejo de ajuda ao outro, motivada por valores altruístas e pela busca do autoconhecimento e de desenvolvimento pessoal. Assim, pode-se pensar que, quando esse precisa atuar também em outra atividade não relacionada à Psicologia, possam ocorrer sentimentos de frustração e desgaste físico e emocional.

0 exercício da profissão em mais de uma área da Psicologia, fortalece a realização profissional, reduz o desgaste, a indiferença frente às pessoas atendidas e o sentimento de não estar atendendo as expectativas sociais atribuídas ao psicólogo. Emery, Wade e McLean ${ }^{14}$ também verificaram que a diversidade de atuação dos psicólogos associa-se ao aumento da realização pessoal com o trabalho. Uma das possíveis razões para entender este resultado é que, o exercício profissional da psicologia nas diversas áreas possa ser percebido pelos profissionais como a possibilidade de promover saúde mental e a qualidade de vida das pessoas para um maior número de pessoas e em variados contextos. Assim, na medida em que isso acorre, aumenta o desejo de atingir os resultados no trabalho realizado, passando este a ser fonte de satisfação.

Em relação ao tipo de vínculo empregatício estabelecido no exercício das atividades laborais dos psicólogos, os resultados sugerem que os profissionais que atuam com vínculo são os que possuem maior desgaste, afastamento emocional da clientela e sentimento de culpa por adotarem comportamentos incompativeis com seu papel profissional. Achados semelhantes foram encontrados nos estudos realizados por Rupert e Morgan ${ }^{17}$, Rupert e Kent ${ }^{15}$ e Rupert, Stevanovi e Hunley. ${ }^{13}$ Tais resultados podem ser entendidos à luz das mudanças ocorridas na profissão do psicólogo. Tradicionalmente, a configuração de trabalho do psicólogo esteve sustentada na atuação autônoma, com predomínio de uma formação sustentada no modelo da prática clínica e do atendimento individual. ${ }^{46,47} \mathrm{Na}$ atualidade, é possível constatar o crescimento da profissão com o fomento das instituições de ensino ${ }^{48} \mathrm{e}$ a assunção de um compromisso de atuação social ${ }^{47}$, o que ampliou a inserção dos profissionais no campo da assistência pública à saúde e em espaços de promoção de saúde coletiva ${ }^{46}$, tendo ocasionado novos vínculos de trabalho. ${ }^{49}$ Nesse sentido, o trabalho autônomo tem compartilhado espaço com a inserção dos profissionais em instituições, especialmente nas do serviço público e de saúde ${ }^{50}$, o que ocasiona a emergência de novos estressores a exemplo da sobrecarga de trabalho, a perda de autonomia ${ }^{17}$ e do controle sobre o trabalho ${ }^{13}$ sendo essas transformações uma das possíveis consequências para que o trabalho assalariado produza maior desgaste profissional que o exercido na modalidade autônoma.

A inserção do profissional em grupos de estudo ou associações aumenta a realização pessoal, reduz o desgaste, a indiferença e a culpabilização. Possivelmente, isso ocorre porque a interação entre os colegas de profissão permite a troca de experiência, favorece o intercâmbio de informações, proporciona a solução de problemas inerentes à prática profissional ${ }^{51} \mathrm{e}$ funciona como recurso para equilibrar elementos da vida profissional e pessoal. ${ }^{15}$ Verifica-se que na medida em que aumentam os ganhos financeiros aumenta o desejo de buscar a consolidação das metas profissionais e diminui o sentimento de exaustão emocional e física, as atitudes de indiferença das pessoas atendidas e a autocobrança em relação ao papel social da profissão. Estudo realizado por Emery, Wade e McLean ${ }^{14}$ com psicólogos corrobora com esses achados, ao identificar que os ganhos financeiros funcionam como um elemento importante da realização profissional. Tais resultados podem estar sinalizando um início de mudança na motivação da escolha da escolha profissional, haja vista ter sido e ainda ser concebida como uma profissão predominantemente influenciada pelo desejo de doação e ajuda ao próximo e pela sobreposição dos valores altruístas aos interesses econômicos. ${ }^{52,49}$ A quantidade de pessoas atendidas diariamente aumenta 0 desgaste físico e emocional dos psicólogos, resultado também encontrado por Rupert, Stevanovic e Hunley ${ }^{13}$ e Roque e Soares ${ }^{53}$ em estudos realizados com psicólogos. Possivelmente, isso ocorre porque as demandas de trabalho desse profissional estão, via de regra, permeadas de alta carga emocional, considerando-se que seus serviços são geralmente solicitados por pessoas que se encontram em sofrimento psíquico.

Resultados obtidos indicam que o aumento da quantidade de carga horária semanal aumenta a ilusão pelo trabalho e a insensibilidade em relação às pessoas atendidas. A relação entre a carga horária e a realização com o trabalho, também foi identificada em estudos com psicólogos americanos. ${ }^{12,13,15,17}$ É possivel supor que quanto maior a carga horária do psicólogo, maior é o envolvimento efetivo com as atividades que realiza e a constatação dos resultados das suas ações, possibilitando perceber seu trabalho como atrativo e fonte de realização pessoal. Por outro lado, os que atendem maior número de pessoas podem ver reduzido o tempo dedicado ao atendimento para dar conta das demandas com implicações para a qualidade do serviço prestado, o que pode explicar o afastamento emocional e a indiferença. ${ }^{54}$

0 aumento da satisfação com a profissão aumenta o desejo de atingir as metas profissionais e diminui as demais dimensões do Burnout. Por outro lado, o aumento do pensamento vinculado a troca da profissão, reduz a motivação para o atingimento de resultados profissionais e aumenta as demais dimensões do Burnout. A satisfação com a profissão está intimamente ligada à satisfação com o trabalho e a SB e o desejo de permanecer na profissão. ${ }^{55}$ Os resultados deste estudo mostram-se condizentes com a literatura que 
aponta para a existência de relação entre a satisfação no trabalho e Burnout. Gil-Monte e Peiró ${ }^{56}$ destacaram que as dimensões de Burnout e satisfação no trabalho estão negativamente relacionadas com os sentimentos de exaustão emocional e despersonalização e positivamente relacionada com a dimensão de realização pessoal no trabalho. Senter et $\mathrm{al}^{18}$ identificaram associação positiva entre satisfação no trabalho e a percepção de competência dos psicólogos.

\section{CONCLUSÃO}

Este estudo, na medida em que identificou a prevalência de Burnout e a associação entre suas dimensões e variáveis sociodemográficas e laborais confirma que a profissão de psicólogo possui risco de adoecimento da síndrome e que está relacionada a fatores individuais e da organização do trabalho. Neste sentido, identificou-se um perfil de risco constituído de homens, profissionais sem companheiro(a) fixo(a), sem filhos, com menores ganhos financeiros, menor carga horária de trabalho semanal e maior número de atendimentos/semanais, que atuam em outra atividade não relacionada à Psicologia ouem apenas uma área da Psicologia, que possuem vínculo empregatício e não participam de grupos de estudo ou associações da Psicologia.

0 estudo apresenta algumas limitações a serem consideradas na leitura de seus resultados: a primeira é o seu delineamento transversal, o que impossibilita a análise de relações causais; a segunda é o tipo de amostra não probabilística, que não permite a generalização de seus resultados; a terceira diz respeito ao caráter regional da amostra investigada, a qual foi selecionada e um único Estado do Brasil, consequentemente, impedindo a generalização de seus resultados para outras regiões; a quarta refere-se a utilização de medidas de autorrelato, que pode ocasionar algum tipo de viés em razão da desejabilidade social que algumas questões abordam. No caso particular deste estudo, podem-se citar as questões relacionadas à dimensão de Indolência, pois pode ser difícil para o psicólogo assumir que se distancia e trata de forma impessoal as pessoas que atendem. Os resultados obtidos sugerem a necessidade de aprofundamento por meio de estudos longitudinais, com amostras probabilísticas e em diferentes estados e regiões do Brasil. Também, pelas especificidades do campo de atuação, recomendam-se análises por tipo de área de atuação. Em relação a intervenções, sugerem-se duas linhas de trabalho. A primeira com ações de prevenção a serem desenvolvidas durante a formação profissional sensibilizando futuros psicólogos para os possiveis fatores de risco de Burnout presentes na profissão e, a segunda voltada aos profissionais para a prevenção dos fatores de risco específicos do seu campo de atuação e para a reabilitação ações voltadas para o manejo de estressores. Para ambas situações, é importante estimular o autodiagnóstico e autocuidado.

\section{REFERENCIAS}

1. Faúndez VEO, Gil-Monte PR. Prevalencia del síndrome de quemarse por El trabajo (burnout) en trabajadores de servicios en Chile. Información Psicológica. 2007; (91-92):43-52

2. Harrison BJ. Are you to burn out? Fund Raising Management. 1999;30(3):25-28.

3. Maslach C. Job burnout. Ann Rev Psychol.2001;52:397-422.

4. Smith PL, Moss B. Psychologist impairment: what is it, how can it be prevented, and what can be done to address it? ClinPsychol-Sci Pr.2009;16(1):1-15.

5. Cebrià-Andreu J. El sindrome de desgaste profesional como problema de salud pública. Gaceta Sanitaria. 2005;19(6):470

6. Carlotto MS. Sindrome de Burnout em professores: prevalência e fatores associados. Psic-Teor e Pesq. 2011;27(4):403-410.

7. Gil-Monte PR. El sindrome de quemarse por El trabajo ("Burnout"). Una enfermedad laboral en la sociedad del bienestar. Madrid: Pirámide;2005.

8. Jacques M. Saúde/Doença no trabalho do psicólogo: a Síndromede Burnout. In: Bastos A,GondinS, organizadores. 0 Trabalho do Psicólogo no Brasil. Porto Alegre: Artmed; 2011. p.338-358.

9. Moreno-Jiménez B. Validez factorial del inventario de burnout de psicólogos en una muestra de psicólogos mexicanos. Rev Latinoam Psicol. 2006;38(3):445-456.

10. Bastos A. As mudanças no exercicio profissional da psicologia no Brasil: o que se alterou nas duas últimas décadas e o que vislumbramos a partir de agora? In: Bastos A,GondinS, organizadores. 0 Trabalho do Psicólogo no Brasil. Porto Alegre: Artmed; 2011. p.419-495.
11. Rupert PA, Baird KA. Managed care and the independent practice of psychology. Prof Psychol-Res Pr.2004;35(2):185-193.

12. Lasalvia A. Influence of perceived organizational factors on job burnout: survey of community mental health staff. J Psychiatr.2009;195:537-544.

13. Rupert PA. Work-family conflict and burnout among practicing psychologists. Prof Psychol-Res Pr.2009;40(1):54-61.

14. Emery S. Associations among therapist beliefs, personal resources and burnout in clinical psychologists. Behav Chang.2009;26(2):83-96.

15. Rupert PA, Kent JS. Gender and work setting differences in career-sustaining behaviors and burnout among professional psychologists. Prof Psychol-Res Pr.2007;38(1):88-96.

16. Rupert PA, et al. Work demands and resources, work-family conflict, and family functioning among practicing psychologists. Prof Psychol-Res Pr.2013;44(5):283-289.

17. Rupert PA, Morgan DJ. Work setting and burnout among professional psychologists. Prof Psychol_Res Pr.2005;36(5):544-550.

18. Senter A. Correctional psychologist burnout, job satisfaction, and life satisfaction. Psychol Serv.2010;7(3):190-201.

19. Maslach C. Making a significant difference with burnout interventions: researcher and practitioner collaboration J Organ Behav. 2012;33:296-300.

20. Ferrari R. Avaliação da síndrome de burnout em profissionais de saúde: uma revisão sistemática da literatura. Revista Eletrônica Gestão \& Saúde. 2012;3(3): 1150-165. 


\section{REFERENCIAS}

21. Carlotto MS. Prevalence and factors associated with burnout syndrome in professionals in basic health units. CiencTrab. 2013;15(47):73-77.

22. Brasil. Ministério da Saúde. Doenças relacionadas ao trabalho: Manual de procedimentos para os serviços de saúde. Brasilia, DF: MS; 2001.

23. Batista JB. Sindrome de Burnout: confronto entre o conhecimento médico e a realidade das fichas médicas. Psicol Estud. 2011;16(3):429-435.

24. Gil-Monte PR. Validation of the Brazilian version of the "Spanish Burnout Inventory" in teachers. RevSaúde Pública.2010;44:140-147.

25. Goodman LA. Snowball sampling. Ann Math Stat.1961;32:148-170.

26. Heckathorn DD. Respondent-driven sampling: a new approach to the study of hidden populations. SocProbl.1997;44(2):174-199.

27. Goel S, Salganik MJ. Respondent-driven sampling as Markov chain Monte Carlo. Stat Med.2009; 28(17):2202-2229.

28. Gil-Monte PR. CESOT. Cuestionario para la evaluacióndel Síndrome de Quemarse por elTrabajo; Manual. Madrid: TEA; 2011.

29. Cohen J. A Power Primer. Psychol Bull. 1992;112(1):155-159.

30. Field AP. Descobrindo a estatistica usando o SPSS. Porto Alegre: Artmed; 2009.

31. Cronbach $\sqcup$, Shavelson RJ. My current thoughts on coefficient alpha and successor procedures. Educ Psychol Measur. 2004;64(3):391-418.

32. Carlotto MS,Palazzo LS. Sindrome de burnout e fatores associados: um estudo epidemiológico com professores. Cad Saúde Pública. 2006;22:1017-1026.

33. Marucco MA. Sindrome de quemarse por El trabajo (burnout) en pediatras de hospitales generales, estudio comparativo de la prevalencia medida com el MBI-HSS y el CESOT. Informació Psicológica. 2008;91(92):32-42.

34. Tejada P, Gómez V. Prevalencia y factores demográficos y laboral associados al burnout de psiquiatras em Colombia. Universitas Psychologica. 2012;11(3): 863-873.

35. Sobral MFC, Lima MEO. Representando as práticas e praticando as representações nos CRAS de Sergipe. Psicol Cienc Prof. 2013;33(3):630-645.

36. Freudenberger HJ, Richenson G. Burn out: how to beat the high cost of success. New York: Bantam Books; 1980.

37. Maslach $C$, Jackson SE. The role of sex and family variables in burnout. Sex Roles.1985; 12(7/8):837-851.

38. Purvanova RK, Muros J P. Gender differences in burnout: a meta-analysis. J VocatBehav. 2010;77(2):168-185.

39. Rohlfs D. La perspectiva de género el estudio de las diferencias y desigualdades en salud [Resumen]. En: Anais de la I Jornada de La Red de Médicas y Profesionales de La Salud, Madrid,1999. Vol. 2. Madrid: RMPS; 1999. p.12-13.

40. Caetano E, Neves CEP. Relações de Gênero e precarização do trabalho docente. Revista HISTEDBR On-Line [online]2009 [citado nov 2014] 9(33e):251-263.
Disponible em: http://www.fe.unicamp.br/revistas/ged/histedbr/article/view/ 4067/3370

41. Negreiros TC, Féres-Carneiro T. Masculino e feminino na familia contemporânea. Est Pesq Psicol. 2004;4(1):34-47.

42. Mazer SM, Melo-Silva LL. Identidade profissional do psicólogo: uma revisão da produção científica no Brasil. Psicol Ciênc Prof.2010; 30(2): 276-95.

43. Bontempo XF. Nivel de síndrome de agotamiento en médicos, enfermeras y paramédicos. RevMexicPuerPed.1999; 6(2): 252-260.

44. Leiter MP, Havie PL. Burnout among mental health workers: a review and a research agenda. Int J SocPsychiatr.1996;42(2): 90-111.

45. Magalhães $M$, et al. Eu quero ajudar as pessoas: a escolha vocacional da psicologia. PsicolCiêncProf. 2001;21(2): 10-27.

46. Dimenstein M. 0 psicólogo eo compromisso social no contexto da saúde coletiva. PsicolEst. 2001; 6(2):57-63.

47. Dutra JS. Competências: conceitos e instrumentos para a gestão de pessoas na empresa moderna. São Paulo: Atlas; 2004.

48. Yamamoto $\mathrm{OH}$. A formação básica, pós-graduada e complementar do psicólogo no Brasil. In: Bastos A,GondinS, organizadores. 0 Trabalho do Psicólogo no Brasil. Porto Alegre: Artmed; 2011. p.45-65.

49. Gondim S. Áreas de atuação, atividades e abordagens teóricas do psicólogo brasileiro. In: Bastos A, Gondin S, organizadores. 0 Trabalho do Psicólogo no Brasil. Porto Alegre: Artmed; 2011.p.174-199.

50. Heloani R. 0 exercício da profissão: caracteristicas gerais da inserção profissional do psicólogo. In: Bastos A, Gondin S, organizadores. 0 Trabalho do Psicólogo no Brasil. Porto Alegre: Artmed; 2011. p.107-130.

51. American Psychological Association. Not going it alone: peer consultation groups [on line]. Washington, DC: APA; 2005[cited nov 2014]. Retrieved from http://www.apapracticecentral.org/ce/self-care/peer-consult.aspx

52. Carvalho AA. Escolha da profissão: alguns valores implicitos nos motivos apontados pelos psicólogos. In: Conselho Federal de Psicologia, organizador. Que é o psicólogo Brasileiro? São Paulo: Edicon; 1988.p.49-68

53. Roque $L$, Soares $L$. Burnout numa amostra de psicólogos portugueses da região autónoma da Madeira. Psic Saúde \& Doenças 2012;13(1): 2-14.

54. Cornelius A, Carlotto MS. Síndrome de Burnout em profissionais de atendimento de urgência. Psicologia em Foco.2007;1(1): 15-27.

55. Shapiro JP, et al. Job satisfaction and burnout in child abuse professionals: measure development, factor analysis, and job characteristics. J Child Sex Abus. 1996;5(3):21-38.

56. Gil-Monte PR, Peiró JM. Desgaste psíquico en el trabajo: el síndrome de quemarse. Madrid: Sintesis; 1997. 